\title{
Rutas criticas de mujeres en situación de violencia familiar. Estudio de casos en la ciudad de Buenos Aires
}

\author{
Critical paths of women in situation of domestic violence. Cases study at Buenos \\ Aires city
}

\section{Claudia Elisabet Teodori ${ }^{1}$}

Resumen: La violencia familiar es una de las tantas manifestaciones de la violencia de género y constituye para las mujeres una de las principales causas de morbimortalidad. A través de un estudio de casos, utilizando registros de admisión y entrevistas a usuarias de un servicio de atención de violencia familiar de un Hospital General de la Ciudad de Buenos Aires, se analiza el proceso recorrido (ruta critica) por mujeres con experiencia de violencia para obtener atención, enfrentar y resolver su problema. Se examinaron las respuestas institucionales, comunitarias y sanitarias según la percepción de las mujeres, atendiendo a la calidad y accesibilidad de recursos. El $80 \%$ sufrió violencia física. El 36\% llegó por recomendación judicial y un 32\% derivadas por servicios del propio hospital. El 78\% habia efectuado denuncia policial. Un tercio inició la ruta crítica por riesgo de sus hijos. $70 \%$ de los agresores fueron parejas varones. Recursos institucionales dispersos e inconexos, el inadecuado accionar policial, la carencia de medios para enfrentar un proceso judicial y la desarticulación intra e interinstitucional, generan re-victimización y recrudecen el padecimiento de las mujeres.

Palabras clave: Género, violencia familiar, salud de las mujeres, servicios sanitarios.

\begin{abstract}
Domestic violence, is one of the many manifestations of gender violence and is a main source of morbidity and mortality for women. Thorough triangulation of methods and sources we used admission records of women at service of domestic violence in a general hospital at Buenos Aires and made interviews. The research aimed to analyze the critical path for women to get attention and face the problem of violence and examine institutional and community responses according to their perception related to the quality and accessibility of resources. The $80 \%$ suffered physical violence. $36 \%$ were referred by judicial recommendation and $32 \%$ derived from services of the hospital itself. $78 \%$ made a police report about the situation of violence. A third of the women started the critical path when a risk situation happened to their children. $70 \%$ of the aggressors were male partners. Institutional resources spread and disjointed, improper police action, the lack of means to stand trial and the intra and inter institutional coordination, generate re-victimization and step up the suffering of women.
\end{abstract}

Keywords: Gender, domestic violence, women's health, health services.

1 Universidad de Buenos Aires, Hospital Gral. de Agudos Dr. T. Álvarez, Ciudad Autónoma de Buenos
Aires, Asoc. Civil DECIDIR. (clauteodori@gmail.com). El presente artículo recoge resultados del trabajo de
investigación "Rutas criticas de mujeres con experiencia de violencia intrafamiliar en la Ciudad de Buenos
Aires. Estudio de casos en usuarias del dispositivo violencia de género del Hospital General de Agudos Dr.
T. Álvarez" que fue desarrollado por la autora con el apoyo del Ministerio de Salud de la Nación Argentina,
a través de una beca otorgada por la Comisión Nacional Salud Investiga. Dicho trabajo es retomado por la
autora en "A los saltos buscando el cielo. Trayectorias de mujeres en situación de violencia familiar" (2015).
Buenos Aires, Editorial Biblos. El protocolo de investigación fue aprobado por el Comité de Ética en
Investigación del Hospital Gral. de Agudos Dr. T. Álvarez. Recibido: 25/03/2015; Aceptado: 29/10/2015. http://dx.doi.org/10.18004/riics.2015.diciembre.179-194 


\section{INTRODUCCIÓN}

La violencia se constituye como problema para el sector salud, a partir del impacto que provoca en la salud de las personas y conjuntos sociales quienes demandan la formulación de políticas específicas y la organización de prácticas y servicios particulares para su abordaje.

En América Latina, donde las violencias representan una de las principales fuentes de morbilidad-mortalidad, la incorporación de la problemática a las agendas de salud de los países se produce a partir de los años 80 en el marco de grandes transformaciones sociales (Minayo, 2005).

En particular, la violencia familiar, responsable de numerosos padecimientos, fue definida por Organización Panamericana de la Salud en 1995 y comprende "toda acción u omisión cometida por algún miembro de la familia en relación de poder, independientemente del espacio físico donde ocurra, que perjudique el bienestar, integridad física, psicológica o la libertad y el derecho al pleno desarrollo de otro(a) miembro de la familia" (Sagot, 2000, p.140). Afecta principalmente a mujeres y niños constituyendo una de las tantas manifestaciones de la violencia de género ya que responde a una desigual distribución de poder (Lamas, 2006). Lejos de constituir un fenómeno de carácter extraordinario o patológico, es producto de la organización social que legitima distintas formas de desigualdad.

En Argentina, se observa una creciente sensibilidad social y politica en torno a este tipo de violencia. Se han creado numerosos programas para su abordaje en distintos sectores y en 2010, se reglamentó la ley de protección integral a las mujeres contra todo tipo de violencia $\left(\mathrm{N}^{\circ}\right.$ 26.485) que en su extenso articulado, recupera la complejidad del problema de las violencias desde una perspectiva de género e involucra de manera pormenorizada a Ministerios Nacionales de: Salud, Educación, Desarrollo social, Justicia Seguridad y Derechos Humanos, Trabajo y Empleo y a la Secretaría de medios de Comunicación (Ley Nº 26.485, 2010).

En 2012, se introdujeron modificaciones al código penal tendientes a resguardar la integridad de las mujeres: eliminación del avenimiento (eximición del violador por contraer matrimonio con la víctima) y se incorporó la figura de femicidio (asesinato de una mujer por razones de género) (Segato, 2011). Sin embargo, el conocimiento acerca de la situación es aún parcial y fragmentado. Para dar cuenta de la magnitud del fenómeno de la violencia contra las mujeres, se 
proyectan cifras de esos asesinatos (proporcionadas por Organizaciones de la Sociedad Civil) o se trabaja con datos de denuncias y consultas recibidas por los servicios de asistencia, por ejemplo informes de la Oficina de Violencia Domestica de la Corte Suprema de Justicia de la Nación (OVD, 2010) o el Consejo Nacional de las Mujeres (CNM, 2008).

Por su parte, estudios que focalizan en los efectores de salud, ponen en evidencia sus dificultades en la visibilización, el abordaje y el proceso de registro de la violencia familiar (Alemán, 2010; Deslandes, 2002). Así, débiles análisis de situación, derivan en el diseño y la ejecución de políticas y prácticas institucionales inadecuadas.

A escala internacional, un estudio multicéntrico realizó aportes sustantivos a la comprensión de la problemática, describiendo los recorridos (rutas críticas) de las mujeres afectadas por la violencia intrafamiliar en América Latina. Los patrones de intervención de los países presentan similitudes: dificultades en los servicios de salud, escasa capacidad de contención de los sistemas judicial, policial y educativo, buena disposición aunque limitada capacidad de las organizaciones comunitarias, falta de articulación intersectorial y escasez de recursos humanos especializados (Sagot, 2000).

A fin de profundizar el conocimiento a nivel local, nuestro problema de investigación se centró en reconstruir los recorridos o "Rutas críticas" de las mujeres afectadas por la violencia familiar abarcando tanto las acciones por ellas emprendidas, como las respuestas institucionales (obstáculos y facilitadores) que encontraron en ese proceso (Sagot, 2000). En los recorridos se exponen aspectos centrales como el conocimiento de los propios derechos, la información disponible y los modos en que sujetos y colectivos diseñan sus propios caminos en función de sus necesidades.

Con el objetivo de analizar el proceso desplegado por las mujeres para obtener atención, enfrentar y resolver su problema, el estudio se desarrolló en el ámbito de un Hospital General de la Ciudad de Buenos Aires por contar con importante casuística y capacidad de motorizar iniciativas intersectoriales. De allí que, el hallazgo de nudos críticos en la respuesta institucional, permitió la elaboración y difusión de recomendaciones para la incorporación transversal de estrategias locales y regionales de prevención, detección y atención de la violencia de género. 


\section{METODOLOGÍA}

Se realizó un estudio de casos con abordaje cuanti- cualitativo de datos, que permitió examinar en profundidad distintos aspectos del fenómeno como los pasos seguidos por las mujeres afectadas en los sistemas de salud y justicia, y el perfil sociodemográfico de las mismas. Se efectuó triangulación de métodos y de fuentes: análisis de registros de atención y entrevistas. El trabajo de campo se desarrolló entre mayo de 2011 y enero de 2012.

Para la dimensión cuantitativa se analizaron registros anuales de ingresos al equipo de Violencia - Salud Mental, entre ellos se seleccionó a todas las mujeres ingresadas, un total de 132. Se describieron variables sociodemográficas (edad, nacionalidad, lugar de residencia) y variables vinculadas a las rutas criticas: tipo de violencia, agresor, forma en que llegó al servicio (espontánea, derivación de salud, judicial, etc.). Previamente se habian unificado criterios para completar los datos que se tomaban de rutina.

Para la instancia cualitativa, en base a una selección intencional, se entrevistó a 17 mujeres que completaron la consulta de admisión, focalizando en las rutas críticas (Comienzo/Acciones subsiguientes/Facilitadores y obstaculizadores/Red social/Respuestas institucionales, etc.). Se lograron examinar rasgos sobresalientes respecto del punto de partida del recorrido relatado, de la presencia de actores significativos, entre otros aspectos.

Del estudio, solo participaron mujeres mayores de 18 años con experiencia de violencia intrafamiliar, que habían iniciado el proceso de atención y prestaron su consentimiento informado. Se realizó una caracterización exhaustiva y actualizada de la oferta institucional para la atención. Los datos de los registros se volcaron a una planilla Excel para el posterior cálculo de frecuencias y elaboración de tablas y gráficos. Las entrevistas fueron grabadas, el material se transcribió, fue codificado y posteriormente categorizado. Para su tratamiento, se utilizó la técnica de análisis de contenido.

\section{RESULTADOS}

Los principales efectores locales, reconocidos en el estudio, dependen de las áreas de Salud, Desarrollo Social y Ministerio Público Fiscal de la Ciudad de Buenos Aires, Corte Suprema de Justicia de la Nación y Ministerio de Justicia y Derechos Humanos de la Nación. 
La institución en la que se desarrolló el estudio cuenta con equipos de Violencia sexual, de Niños/as en situación de vulnerabilidad y de Violencia familiar.

El Equipo de Violencia familiar, dependiente de la División Salud Mental y compuesto por 8 profesionales, durante el año 2011 recibió 230 pacientes adultos que llegaron derivados por diferentes instituciones y en menor medida por demanda espontánea.

Del total de mujeres registradas que recurrieron al Equipo de Violencia ( $\mathrm{n}=$ 132), el $73 \%$ era de origen argentino y su edad promedio de 36.3 años $(18,68)$. La mitad, refirió residir dentro del área de responsabilidad programática del Hospital, mientras que el 15\% residía en el conurbano bonaerense.

En más del $80 \%$ de los casos con datos ( $n=65)$, las consultantes relataron sufrir o haber sufrido violencia física (incluido sometimiento sexual). Más del 70\% de los agresores fueron las parejas o ex parejas varones (uniones formales e informales, convivientes o no convivientes, etc.) (Figura 1).

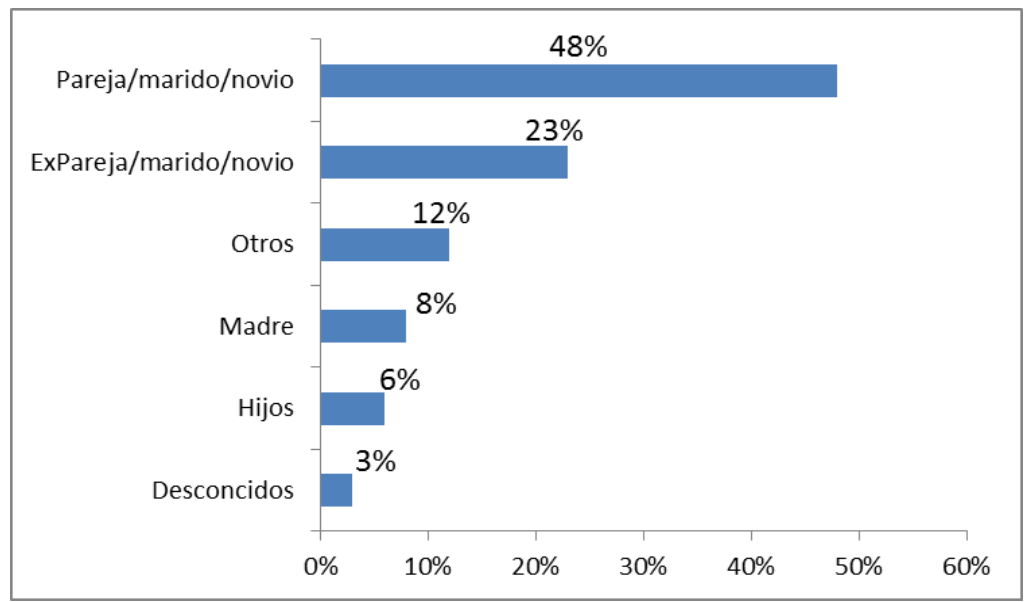

Figura 1. Equipo de violencia, admisiones de mujeres por tipo de agresores, total de 115 casos con datos. Elaboración propia, 2011, en base a datos recolectados por Equipo de Violencia.

Respecto al proceso recorrido (rutas críticas), se relevó la modalidad de acceso al servicio: un $36 \%$ de las mujeres, concurrieron al Equipo de Violencia por 
recomendación judicial o por indicación de defensorías de niños, niñas y adolescentes; derivadas por servicios del propio hospital, llegaron $32 \%$ de las mujeres y de otros hospitales (generales, de rehabilitación y psiquiátricos) provino el 6\% (Figura 2).

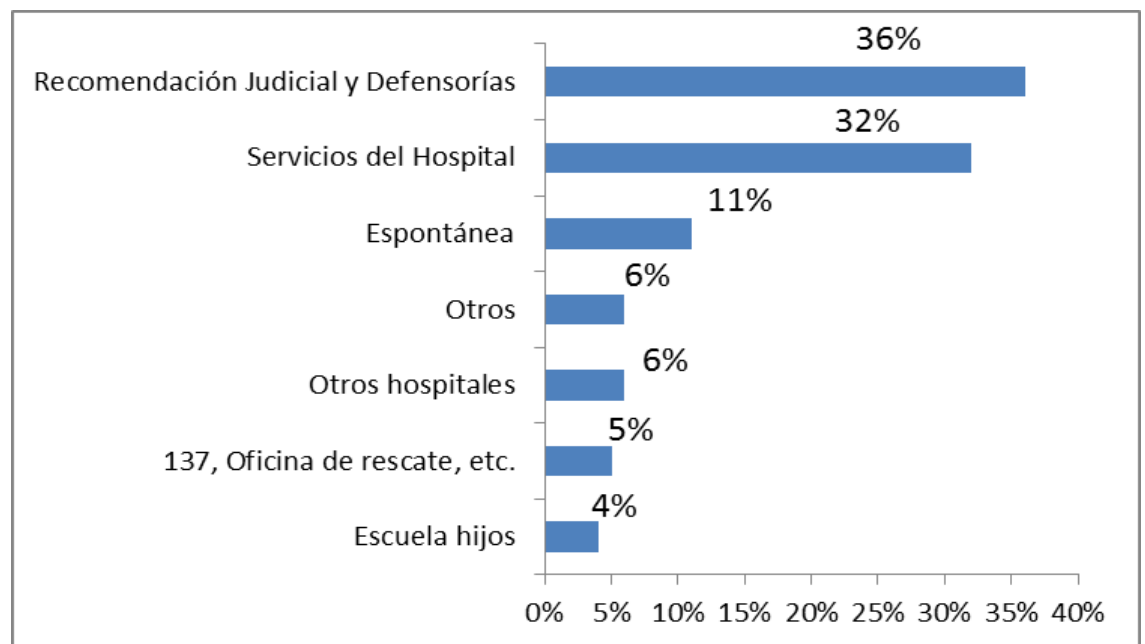

Figura 2. Equipo de Violencia- Admisiones de mujeres según derivación, total de 129 casos con datos. Elaboración propia, 2011, en base a datos recolectados por Equipo de Violencia.

El $60 \%$, refirió haber realizado un tratamiento psicológico y/o psiquiátrico con anterioridad. Las mujeres entrevistadas $(n=17)$, presentaban un perfil similar al del total registrado en la instancia de admisión. Con excepción de una, todas las entrevistadas tenían al menos un hijo y en más del $80 \%$ de los casos, sus agresores fueron las parejas o ex parejas varones. El porcentaje de las mujeres entrevistadas que manifestó sufrir violencia física y sexual ascendió a 90\%, (cifra superior a la del total de los registros, situación que podría deberse al uso de la técnica de entrevista). En relación al nivel de escolaridad alcanzado, 6 de las entrevistadas habian cursado estudios primarios (en forma completa e incompleta), 5 contaban con estudios secundarios y 6 , con estudios terciarios o universitarios (la mitad de las cuales había llegado a completarlos) (Tabla 1). 
Tabla 1. Características de las mujeres entrevistadas.

\begin{tabular}{|c|c|c|c|}
\hline Variable & Valor & Total & Porcentaje \\
\hline \multicolumn{2}{|l|}{ Mujeres entrevistadas } & 17 & $100 \%$ \\
\hline \multirow{7}{*}{ Edad } & hasta 25 años & 5 & $29 \%$ \\
\hline & 26 a 35 años & 4 & $24 \%$ \\
\hline & 36 a 45 años & 3 & $18 \%$ \\
\hline & 46 a 55 años & 3 & $18 \%$ \\
\hline & 56 a 65 años & 0 & 0 \\
\hline & 66 a 68 años & 2 & $12 \%$ \\
\hline & Promedio & \multicolumn{2}{|c|}{36,64 años } \\
\hline \multirow{6}{*}{ Situación laboral } & No trabaja fuera de su casa & 5 & $29 \%$ \\
\hline & No trabaja/ es estudiante & 2 & $12 \%$ \\
\hline & Trabaja como profesional & 2 & $12 \%$ \\
\hline & Trabaja por cuenta propia & 1 & $6 \%$ \\
\hline & $\begin{array}{l}\text { Trabaja en relación de dependencia (no } \\
\text { profesional) }\end{array}$ & 6 & $35 \%$ \\
\hline & Trabaja en empresa familiar & 1 & $6 \%$ \\
\hline \multirow{3}{*}{ Tipo de violencia } & Solo Psicológica y/o verbal & 1 & $6 \%$ \\
\hline & Física y Psicológica & 13 & $81 \%$ \\
\hline & Sexual & 1 & $6 \%$ \\
\hline \multirow{2}{*}{$\begin{array}{l}\text { Tratamiento Psicol. } \\
\text { o psiquiátrico }\end{array}$} & Realizó & 8 & $47 \%$ \\
\hline & No realizó & 9 & $53 \%$ \\
\hline \multirow{4}{*}{ Fue derivada por } & $\begin{array}{l}\text { Servicios de salud y profesionales de la } \\
\text { salud }\end{array}$ & 7 & $41 \%$ \\
\hline & Recomendación judicial & 6 & $35 \%$ \\
\hline & Espontánea & 1 & $6 \%$ \\
\hline & $\begin{array}{l}\text { Otro (Dirección de la mujer, programas de } \\
\text { D. Social de CABA) }\end{array}$ & 3 & $18 \%$ \\
\hline \multirow{2}{*}{$\begin{array}{c}\text { Intervención } \\
\text { policial y / o } \\
\text { judicial }\end{array}$} & Realizó denuncia & 13 & $81 \%$ \\
\hline & No realizó & 4 & $19 \%$ \\
\hline
\end{tabular}

Elaboración propia a partir de datos relevados en entrevistas, 2011. 
Respecto a la modalidad de acceso al servicio, solo una de las mujeres, refirió haber llegado al equipo por demanda espontánea, una joven sexualmente abusada (ASI), quien había accedido a tratamiento luego de 11 años. La tercera parte, llegó, por recomendación judicial y en tres casos por derivación del servicio de emergencia de la institución.

Para dos de las entrevistadas, el contacto personal o de un familiar con profesionales de un servicio del Hospital, contribuyó a que pudieran iniciar un tratamiento. Otras tres entrevistadas, las más jóvenes, destacaron el protagonismo del padre al momento de iniciar el recorrido:

"...la primer denuncia que yo le hago por el tema de que me voy, fui con mi papá, porque yo me fui a la casa de ellos” (D 21 años, un hijo, empleada, violencia psicológica y física, agresor: pareja).

En el caso de la joven víctima de ASI, la referencia a una figura familiar resultó ambigua:

"Mi abuelo me contenía y me decía que denunciar era muy largo y a veces no valía la pena". (D 22 años, sin hijos, estudiante universitaria; agresor: familiar).

En un tercio de los casos, el momento de inicio de la ruta crítica, fue motorizado por una situación de riesgo de sus hijos. Tal es el caso de una mujer, que concurrió al equipo por recomendación de la psicoterapeuta que atendía a su hija (con graves problemas de salud asociados a la situación de violencia) ( $\mathrm{R} 50$ años, hija de 13 años, empresa familiar, violencia psicológica, agresor: marido).

A través de las entrevistas, en los recorridos relatados, se captaron los pasos seguidos por las mujeres y se reconstruyeron los itinerarios en forma gráfica (Figura 3).

Al analizar los recorridos se ubicaron obstáculos de orden personal, como la consideración positiva del agresor "(Él) es una buena persona" (B 24 años, 2 hijos, escolaridad secundaria incompleta, ama de casa, violencia psicológica y física; agresor: pareja) o "...es un buen padre" (R 51 años), la dependencia afectiva "Me cuesta vivir sin mi marido, me crié con el... me enseñó todo..." (L 40 años) o “...prefiero los golpes a que esté con otra” (B 24 años). Algunas entrevistadas, se responsabilizaban por la violencia sufrida “...yo no tenía personalidad..." (R 50 
años) o expresaban sentimientos de culpabilidad: "me sentía culpable por separar a la familia” (D 22 años).

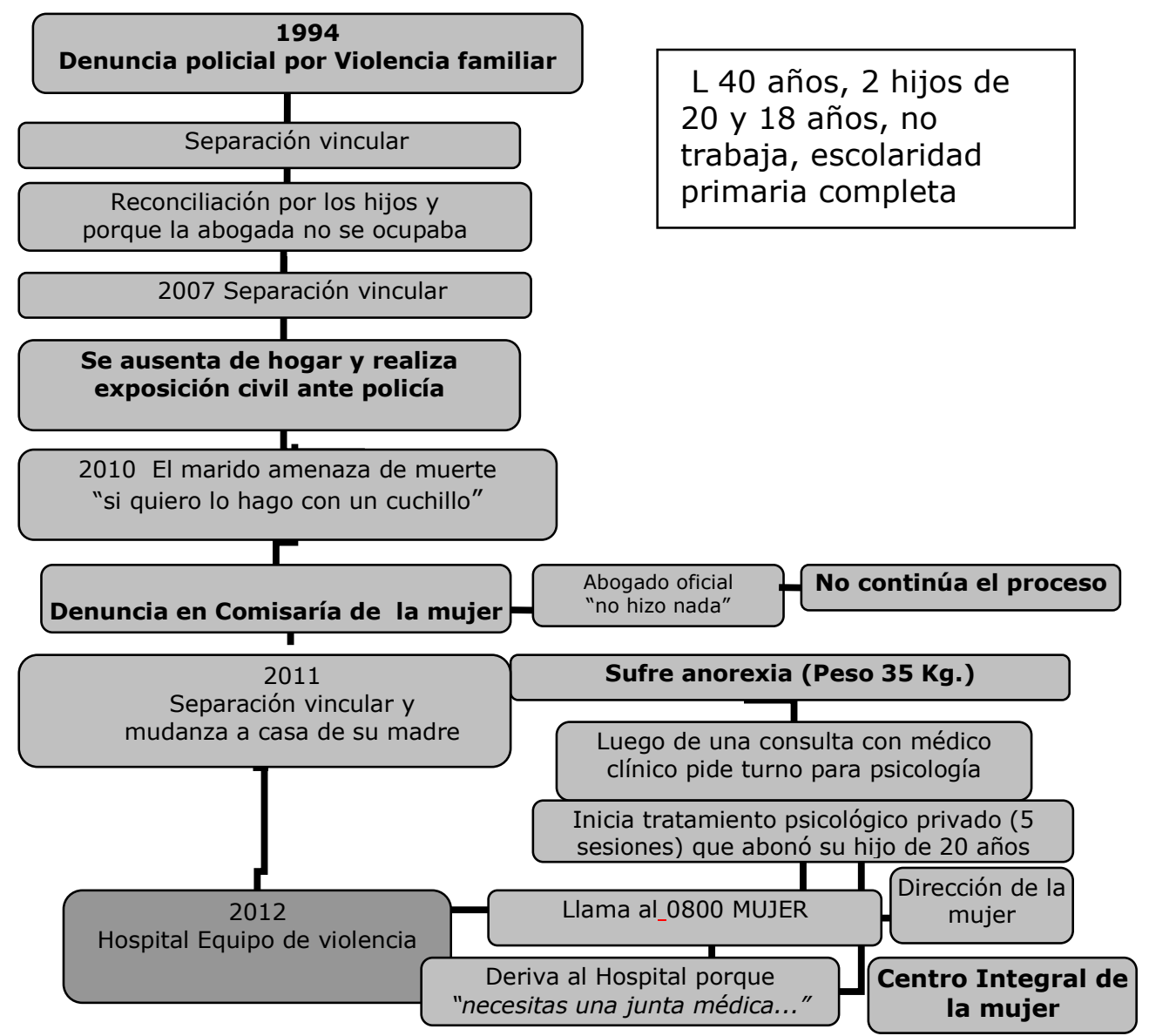

Figura 3. Representación gráfica de los itinerarios relatados por las mujeres entrevistadas. Entrevistada $\mathrm{N}^{\circ} 9$.

La mitad de las entrevistadas, refirió dificultades económicas y más de la tercera parte, señaló que problemas habitacionales coartaron su decisión de poner fin al vínculo violento. Una de ellas, nunca denunció la situación de violencia “...porque iba a tener que irme de su casa” (R 43 años). 
En algunos casos, el capital simbólico de las entrevistadas, fue uno de los facilitadores para el acceso a recursos de asistencia (profesionales, Internet, contacto con otros dispositivos, etc.). La disponibilidad de recursos económicos resultó crucial ante la urgencia de alejarse del hogar con sus hijos. Una de las entrevistadas reveló que debido a que “...tenía la tarjeta” logró alojarse en un hotel en lugar de ir a un refugio para mujeres ( $\mathrm{N} 47$ años, dos hijos jóvenes, empleada, escolaridad primaria, violencia psicológica y fisica, agresor: marido).

La mayoria de las entrevistadas detallaron importantes limitaciones para la resolución de su problema: "No tengo información, (...) Al no tener recursos, me dan un abogado por (...) la escuela de abogados...Y yo no puedo tener contacto con el abogado...” (D 21 años).

Los servicios de salud, aparecen en los relatos de todas las entrevistadas. Una de ellas, refiere que debió recurrir a un medico clínico por dolores producto de una lesión que le había ocasionado su marido y no consiguió que en la primera consulta la revisaran ni le indicaran estudios complementarios.

Varias de las entrevistadas se enfrentaron a profesionales que desestimaron la peligrosidad de sus agresores o desacreditaron sus palabras:

“...la psicóloga (de un centro de la Ciudad de Buenos Aires) me decía que mi marido no era tan agresivo... ¿es que tengo que venir toda golpeada para que me den bolilla?"(L 40 años).

Pero también, hubo dos casos en los que las mujeres destacaron el rol de un profesional médico, una de ellas, que realizaba exámenes prequirúrgicos, señaló "El Dr. (médico traumatólogo) me dice que no me tengo que dejar pegar" (R 28 años, discapacidad motriz, un hijo pequeño, escolaridad primaria incompleta, desocupada, violencia fisica, agresor: ex pareja).

Tres de las entrevistadas, habian realizado algún tratamiento psicológico y cinco un tratamiento psiquiátrico (cuatro de ellas lo estaban cursando al momento de la entrevista). Una de las mujeres quien había sido tratada por un psiquiatra (particular) que atendía a su marido planteó que pese a su disconformidad, se hallaba recluida y "dopada". Otro de los aspectos ligados a la presencia de tratamientos psiquiátricos fue la estigmatización "me recalcaba que era una enferma porque iba al psiquiatra" (R 43 años). 
Respecto a las redes sociales, en sus relatos, las mujeres en general mencionan situaciones de soledad "se borraron todos, no querian saber nada... cuando es un tema de la ley, se alejan" (N 47 años) o situaciones de aislamiento ligadas a prácticas de control de los agresores "Desde que me casé nunca tuve amigos ni me dejó estudiar la secundaria” (L 40 años) o “... por él (agresor) dejé amigos y estudio" (B 24 años).

En otros casos se observa que los vínculos familiares se tornaron en obstáculos para salir de la situación de violencia "...me decía que tenga cuidado, que no lo haga poner mal" (en referencia a una recomendación familiar respecto al agresor) (R 50 años).

En relación a la intervención policial y judicial, dos de las mujeres que nunca habian efectuado denuncias, coincidentemente convivian con su agresor desde hacía más de 13 años y en ambos casos, se hallaban en tratamiento psiquiátrico. En el caso de la joven víctima de ASI tampoco se realizó denuncia, su madre no quiso accionar contra el agresor y en cambio, "...fueron a hablar con él" (D 22 años).

Cerca del $80 \%$ de las entrevistadas había efectuado al menos una denuncia policial. En todos los casos, se trató de violencia ejercida por la pareja.

En torno de esa experiencia, la mayoría señaló comportamientos inapropiados por parte de los funcionarios policiales. Frente a intentos de denunciar violencia en el conurbano bonaerense, dos entrevistadas, solo lograron efectuar una exposición civil.

Respecto a la Oficina de Violencia Doméstica de la Corte Suprema de Justicia de la Nación, se reconoció su rol faciltador del acceso al sistema de justicia valorando positivamente la extensión horaria, la disposición del personal, tanto como el trabajo interdisciplinario. Sin embargo, se criticó el alcance de las intervenciones judiciales promovidas, el perfil de los casos a los que estaría dirigido el servicio, el límite de su jurisdicción (Ciudad de Buenos Aires) y el tiempo de espera.

En numerosas oportunidades, las entrevistadas cuestionaron procedimientos judiciales por inadecuados o improcedentes y también mencionaron medidas judiciales que directamente colisionaban con los postulados de la nueva legislación en la materia a saber, la citación a "las partes" a una 
instancia de mediación (mecanismo de intervención totalmente prohibido en casos de violencia de género por la ley 26.485).

En general, las entrevistadas conocian y habian recurrido a diferentes prestadores, sin embargo, la evaluación que hacian era negativa. Las respuestas institucionales resultaban insuficientes o directamente ineficaces:

"En la realidad no sirve. Si bien capaz que te dan un turno dentro de todo rápido, la abogada nunca, absolutamente sirvió nada. Porque es una. (...) para 500 casos por decir un número" (N 24 años), "Nunca conseguí. Ni en defensoría de menores ni en defensoría de la mujer, tampoco. No conseguí nada" (N 38 años).

\section{DISCUSIÓN}

Una nueva ley nacional aborda las violencias contra las mujeres con perspectiva de género, se ha ampliado la sensibilidad social y se han incrementado los servicios de atención a las violencias, sin embargo consideramos que siguen existiendo grandes obstáculos que se interponen entre las mujeres afectadas y la resolución de sus problemas.

El momento en que las mujeres comunicaron que atravesaban una situación de violencia- considerado el punto de partida de la ruta crítica- es un aspecto clave para comprender los modos de enfrentar la/s violencia/s (Sagot, 2000).

En ese sentido la actuación de las redes sociales y familiares como indican otros estudios, resultó ambigua (Ramírez et al, 2005).

El deterioro que se observa en la salud de algunas de las entrevistadas es notable, aún en aquellas que han mantenido numerosos contactos con prestadores de salud por problemas propios o de sus hijos (anorexia, idea-suicida, accidentes, ataques de pánico, etc.). Los prestadores de salud, aparecieron en general, velando las experiencias de padecimiento (Cortés, 1997), minimizando la situación de riesgo o contribuyendo directamente a la medicalización de la experiencia (Recoder, 2011).

Si bien se ha progresado en el acceso a la justicia, se registran innumerables obstáculos en el desarrollo de los extensos procesos judiciales: recursos de asesoramiento y/o patrocino legal insuficientes e inadecuados, sobrecarga para la 
mujer, intervenciones de funcionarios judiciales sin perspectiva de género o violatorias de los DDHH y/o de la legislación vigente en la materia. Al igual que en otras regiones, como menciona Sagot (2000), la atención policial a víctimas y el manejo de situaciones críticas es deficiente y no se garantizan las medidas de protección para las mujeres y sus hijos como es el caso de la exclusión perimetral del agresor. En ocasiones, el personal policial no toma las denuncias, maltrata y/o desalienta a las mujeres que se acercan a las dependencias policiales, incluidas las Comisarias de la Mujer (supuestamente mas compenetradas con la perspectiva de género).

En cuanto a los recursos para la asistencia de la problemática de la violencia de género en la Ciudad de Buenos Aires, podría decirse que son numerosos pero, coincidentemente con otros estudios de América Latina (Sagot, 2000; Menenghel, 2007), se encuentran dispersos y desarticulados. En las experiencias relatadas se observa un recrudecimiento de los padecimientos atribuible a la escasa coordinación de las instituciones y el desconocimiento mutuo de sus diferentes funciones.

Se pudo constatar que gran parte de los prestadores que participaron de las rutas criticas estudiadas, carecian de protocolos para evaluar riesgo y elaborar planes de emergencia para la protección de las usuarias o realizar derivaciones.

Se advirtió que la accesibilidad de las mujeres a los dispositivos de atención, se vio obstaculizada por estructuras burocráticas y falta de comunicación. Ante la heterogeneidad de los recursos institucionales intervinientes (diferentes jurisdicciones, dependencias, etc.), se torna imprescindible el desarrollo en forma sistemática de acciones intersectoriales de articulación.

De la presente investigación, se desprende que la demanda espontánea en el dispositivo estudiado, es muy baja (11\%). No obstante, en un número significativo de recorridos relatados se identificaron momentos de búsqueda de atención que podrían ser considerados de demanda espontánea, los cuales finalmente sólo se tradujeron en oportunidades perdidas.

De aquí, se colige la necesidad de garantizar atención y asesoramiento integrales en las diversas instancias del proceso apelando al trabajo interdisciplinario y a una adecuada coordinación intra/interinstitucional e intersectorial. 
Es de esperar que la progresiva implementación de dispositivos "sensibles a la violencia de género" y la difusión de información y recursos para su atención, contribuyan a incrementar la consulta temprana espontánea.

Respecto a la evolución de las experiencias de violencia, y su temprana iniciación en la etapa de la adolescencia, resulta de vital importancia impulsar estrategias de prevención de noviazgos violentos y violencia de género entre jóvenes.

En cuanto a los prestadores de salud, estos se constituyen en piezas clave para el proceso de prevención, detección y atención de la violencia familiar y sus consecuencias. Su capacitación entonces, debiera proporcionar elementos para:

- el manejo de indicadores para la detección de maltrato y/o abuso,

- establecer relaciones entre determinadas patologías y situaciones de victimización,

- combatir mitos y estereotipos de género que obstaculizan el abordaje de muchos problemas de salud/ enfermedad en mujeres,

- conocer la legislación vigente,

- documentar sistemáticamente los casos,

- manejar los recursos disponibles para realizar derivaciones cuidadas,

- efectuar evaluación de riesgo de vida y de salud integral,

- de ser necesario, elaborar plan de seguridad junto a las mujeres para su protección,

- realizar seguimiento.

Para ello, se requiere en muchos casos, un re-diseño institucional. Por último, como observamos, la sistematización y centralización de datos acerca de la violencia de género, es exigua y a pesar de algunas iniciativas, no alcanza para efectuar contribuciones que mejoren su abordaje. En el campo de la salud, se torna imprescindible que la investigación focalice en los tópicos de violencia, género y trayectorias de atención. Conocimientos fundados y rigurosos aportarán a la optimización de los procesos de atención, dando sustento a las prácticas institucionales y a las políticas públicas.

Sería recomendable avanzar en investigaciones que tiendan a:

- establecer prevalencia de violencias en poblaciones de mujeres y describir su perfil, 
- documentar y cuantificar los casos que consultan en busca de atención en los diferentes sectores,

- evaluar impacto de las intervenciones de los dispositivos de atención,

- explorar la relación entre criterios diagnósticos y experiencias de victimización,

- actualizar información sobre los dispositivos de atención,

- rastrear los obstáculos que se presentan para las usuarias y los propios prestadores.

Para atender a las cuestiones antes planteadas, se requiere que el sector salud le otorgue a la problemática de la violencia, la jerarquía de verdadero problema de salud pública.

\section{AGRADECIMIENTOS}

A la Dra. María Laura Recoder, Dra. Diana Galimberti y Comité de violencia del Hospital General de Agudos Dr. T. Álvarez

\section{REFERENCIAS}

Alemán, M. (2010). Detección de violencia basada en género: Análisis de situación del sistema de atención primaria de salud en ciudad de Buenos Aires. Revista Argentina de Salud Pública, 1(5):22-27.

CNM (Consejo Nacional de las Mujeres). (2008). $3^{\circ}$ Informe de evaluación de resultados información producida por servicios usuarios del instrumento de registro de casos de violencia familiar. Recuperado de: disponible en: www.cnm.gov.ar

Cortés, B. (1997). Experiencia de enfermedad y narración: El malentendido de la cura. Nueva Antropología, XVI, 52.

Deslandes, S. (2002). Frageis deuses: Profissionais da emergencia entre os danos da violencia e a recriaçao da vida. Río de Janeiro: Editora Fiocruz.

Lamas, M. (2006). La perspectiva de género en material de formadores en educación sexual y prevención del vih/sida. Buenos Aires: Ministerio de Educación de la Nación.

Ley $\mathrm{N}^{\circ}$ 26.485. (2010). Ley de protección integral para prevenir, sancionar y erradicar la violencia contra las mujeres en los ámbitos en que desarrollen sus relaciones interpersonales. Recuperado de: www.infoleg.gov.ar/infolegInternet/anexos/ $150000 \ldots /$ norma.htm 
Menenghel, S. (2007). Rotas criticas mulheres enfrentando a violência. Sao Leopoldo: Editora Unisinos.

Minayo, M. (2005). Relaciones entre procesos sociales, violencia y calidad de vida. Salud Colectiva, 1(1):69-78.

OVD (Oficina de Violencia Doméstica). (2010). Presentación estadísticas 5 de noviembre de 2009. Recuperado de: www.csjn.gov.ar/docus/documentos/cons_temaovd.jsp

Ramírez, C.; Valdez Santiago, R., \& Hernández Rosete, D. (2005). La percepción del apoyo social en mujeres con experiencia de violencia conyugal. Revista de Salud Mental Instituto Nacional de Psiquiatría Ramón de la Fuente, 28(4):66-73.

Recoder, L. (2011). Vivir con hiv-sida: Notas etnográficas sobre el mundo de la enfermedad y sus cuidados. Buenos Aires: Editorial Biblos.

Sagot, M. (2000). La ruta critica de las mujeres afectadas por la violencia intrafamiliar en América Latina. Washington: OPS.

Segato, R. (2012). Femigenocidio y feminicidio: Una propuesta de tipificación. Recuperado de: http:/ /www.herramienta.com.ar/revista-herramienta-n-49/femigenocidio-y-feminicidiouna-propuesta-de-tipificacion

Teodori, C. (2015). A los saltos buscando el cielo: Trayectorias de mujeres en situación de violencia familiar. Buenos Aires: Editorial Biblos. 